\title{
THE EFFICACY OF PECS 2 BLOCK FOLLOWING RADICAL MASTECTOMY WITH MULTIMODAL ANALGESIC TREATMENT : A RANDOMISED, CONTROLLED, TRIPLE-BLINDED TRIAL.
}

\author{
Aboud Al Ja'bari, ${ }^{1}$ Moira Baeriswyl,2 Kariem El-Boghdadly,3 Eric Albrecht4 \\ 1 Fellow 2 Consultant, 4 Program Director of Regional Anaesthesia, Department of Anaesthesia, Lausanne University Hospital, Lausanne, \\ Switzerland; 3 Consultant, Department of Anaesthesia, Guy's \& St Thomas' NHS Foundation Trust, London, United Kingdom.
}

\section{Background}

- The pectoral nerves 2 (PECS2) block is a regional anaesthetic technique developed to relieve pain after breast surgery, but efficacy data following radical mastectomy are scarce.

- This randomised controlled triple-blinded trial tested the hypothesis that PECS2 block would provide effective analgesia after radical mastectomy.

\section{Methods}

- Fifty patients scheduled for radical mastectomy under general anaesthesia were randomly allocated either to receive a PECS2 block or receive no block, after induction of general anaesthesia.

- Perioperative multimodal analgesic treatment consisted of dexamethasone, magnesium, paracetamol, ibuprofen and intravenous morphine patient-controlled analgesia, following a pre-defined protocol.

- The primary outcome was iv morphine consumption at $24 \mathrm{~h}$.

- Secondary outcomes were iv morphine consumption at 2 and 48 hours, visual analogue pain scores at rest at 2, 24 and 48 hours, presence of postoperative nausea and vomiting and pruritus at 24 and 48 hours, and presence of chronic pain at 6 months.

\section{Results}

- Demographic data were equivalent in both groups (table 1 ).

- Iv morphine consumption at 24 postoperative hours was significantly reduced in the PECS2 group $(5.0 \mathrm{mg}, 95 \% \mathrm{Cl}$ : 2.5-7.5) compared to the control group $(9.7 \mathrm{mg}, 95 \% \mathrm{Cl}$ : 5.8-13.7, $p=0.04)$.

- The secondary pain outcomes were also similar between groups (table 2), with the exception for intravenous morphine consumption at 48 postoperative hours in favour of the PECS2 group.
Table 1. Patient characteristics and clinical data of patients who received pectoral nerves (PECS) 2 blocks and control patients who did not. Data are presented as mean $(95 \% \mathrm{Cl})$ or number as appropriate. $\mathrm{P}<0.05$ is statistically significant.

\begin{tabular}{lccc}
\hline & $\begin{array}{c}\text { PECS 2 } \\
(\mathbf{n}=\mathbf{2 0})\end{array}$ & $\begin{array}{c}\text { Control } \\
(\mathbf{n}=\mathbf{2 2})\end{array}$ & p value \\
\hline Age (y) & $55(49-60)$ & $54(47-61)$ & 0.87 \\
Height (cm) & $167(163-170)$ & $164(161-166)$ & 0.13 \\
Weight (kg) & $66(61-71)$ & $74(64-84)$ & 0.15 \\
Body mass index $\left(\mathbf{k g . m}^{-2}\right)$ & $23.8(22.0-25.7)$ & $27.6(24.2-30.9)$ & 0.05 \\
$\begin{array}{l}\text { ASA (1 / 2 / 3) } \\
\text { Duration of surgery (min) }\end{array}$ & $159(137-183)$ & $146(127-165)$ & 0.34 \\
$\begin{array}{l}\text { Duration of anaesthesia } \\
\text { (min) }\end{array}$ & $205(182-228)$ & $195(174-216)$ & 0.52 \\
\hline
\end{tabular}

Table 2. Secondary pain outcomes of patients who received pectoral nerves (PECS) 2 blocks and control patients who did not. Data are presented as mean $(95 \% \mathrm{Cl})$ or number (\%), as appropriate. Pain scores are reported at rest on the visual analogue scale of 0-10. Patient satisfaction is reported on a visual analogue scale of $0-10 . P<0.05$ is statistically

\begin{tabular}{lccc}
\hline significant. & $\begin{array}{c}\text { PECS 2 } \\
(\mathbf{n}=\mathbf{2 0})\end{array}$ & $\begin{array}{c}\text { Control group } \\
(\mathbf{n}=\mathbf{2 2})\end{array}$ & p value \\
\hline 2 postoperative hours & & & \\
$\quad$ Pain score & $1.6(0.7-2.4)$ & $2.5(1.5-3.5)$ & 0.16 \\
$\begin{array}{l}\text { i.v. morphine consumption } \\
\text { (mg) }\end{array}$ & $1.5(0.0-3.1)$ & $3.8(1.9-5.7)$ & 0.06 \\
$\begin{array}{l}\text { PONV } \\
\text { Pruritus }\end{array}$ & $1(5 \%)$ & $0(0 \%)$ & 0.29 \\
\hline $\begin{array}{l}\text { 24 postoperative hours } \\
\text { Pain score }\end{array}$ & $0(0 \%)$ & $0(0 \%)$ & N/A \\
$\begin{array}{l}\text { PONV } \\
\text { Pruritus }\end{array}$ & $0.8(0.2-1.5)$ & $1.2(0.4-2.2)$ & 0.39 \\
\hline $\begin{array}{l}\text { 48 postoperative hours } \\
\text { Pain score }\end{array}$ & $2(10 \%)$ & $2(9 \%)$ & 0.92 \\
$\begin{array}{l}\text { i.v. morphine consumption } \\
\text { (mg) } \\
\text { PONV }\end{array}$ & $0(0 \%)$ & $1(5 \%)$ & 0.33 \\
\hline $\begin{array}{l}\text { Pruritus } \\
\text { Patient satisfaction }\end{array}$ & $0.2(0.0-0.4)$ & $0.9(0.1-1.7)$ & 0.09 \\
\hline
\end{tabular}

\section{Conclusions}

- The PECS 2 block provides marginal postoperative analgesia in the setting of perioperative multimodal analgesic treatment after radical mastectomy, without impact on chronic postoperative pain. 